\title{
Implantação de um canteiro medicinal didático em uma Unidade Básica de Saúde do Distrito Federal: relato de experiência
}

\author{
Implementation of a didactic medicinal garden in the Basic Health Unit - Distrito \\ Federal: experience report
}

Yasmine Pinto Cabral Galante ${ }^{1}$, Fernando Henrique Aires² ${ }^{2}$ Lígia Villela Rodrigues ${ }^{3}$, Ilze Kaippert ${ }^{4}$, Juliana de Souza Lapa ${ }^{5}$, Rodolfo Rego Deusdará Rodrigues ${ }^{6}$

\author{
1 Universidade de Brasília (UnB), Brasil. E-mail: cabral.yasmine@gmail.com \\ 2 Secretaria de Saúde do Distrito Federal. E-mail: fernandohaires@gmail.com \\ 3 Universidade de Brasília (UnB), Brasil. E-mail: ligiavrodrigues@gmail.com \\ 4 Secretaria de Saúde do Distrito Federal. E-mail: ilzekaippert@yahoo.com.br \\ 5 Universidade de Brasília (UnB), Brasil. E-mail: julianaslapa@gmail.com \\ 6 Universidade de Brasília (UnB), Brasil. E-mail: rodolfodeusdara@gmail.com
}

Recebido em: 02/06/2020 | Aprovado em: 24/07/2020

DOI: $10.12957 /$ interag.2019.53551

\section{Resumo}

O uso das plantas para tratamento de enfermidades é uma prática comum no Brasil. No entanto, observa-se uma carência de profissionais capacitados para orientar o uso racional e seguro de espécies medicinais à população. Considerando a importância de ampliar o conhecimento sobre as plantas medicinais nos profissionais de saúde e comunidade adstrita, o estudo tem como objetivo relatar a experiência da implantação de um canteiro medicinal didático em uma unidade básica de saúde do Distrito Federal. Inicialmente, foi criado um grupo composto por uma residente em medicina de família e comunidade, uma terapeuta ocupacional e uma assistente social pertencentes ao núcleo de apoio à saúde da família. A implantação do canteiro foi realizada em quatro encontros os participantes do projeto, onde foi trabalhado o uso seguro e racional das plantas medicinais, além do cultivo, manejo e plantio das espécies estudadas no canteiro medicinal didático. A criação do canteiro medicinal proporcionou um ambiental ideal para a discussão de temas relacionados ao autocuidado, além disso, promoveu a capacitação dos profissionais neste assunto e evidenciou a carência por programas e modelos que versem sobre o tema.

Palavras-chave: Plantas medicinais, Atenção Primária a Saúde, Sistema Único de Saúde.

\begin{abstract}
The use of medicinal plants is an ancient practice and is widespread in various cultures around the world. In Brazil, the use of plants to treat diseases is very common and is part of the country's popular culture. However, there is a shortage of trained professionals to guide the rational and safe use of medicinal species for the population. Thus, the objective of this work was to report the experience of implementing a Didactic Medicinal Site in a Basic Health Unit in the Distrito Federal, in order to expand the knowledge about medicinal plants among health professionals and the registered community. A group was created consisting of a resident in Family and Community Medicine, an occupational therapist and a social worker belonging to the local Family Health Support Center (NASF). Four meetings were held with the project participants, where the safe and rational use of medicinal plants was studied and researched, in addition to the cultivation, management and planting of the species studied in the didactic medicinal site. At the end of the project, it was possible to conclude that the creation of a medicinal garden provided an ideal environment for the discussion of topics related to self-care, in addition to promoting the training of professionals in the area of medicinal plants and evidencing the lack of programs and models that deal with of the same subject.
\end{abstract}

Keywords: Medicinal plants, Primary Health Care, Unified Health System 
Área temática: Saúde.

Linha de extensão: Saúde e educação.

\section{Introdução}

O uso das plantas medicinais é de longa data uma prática bem difundida em todo o mundo, contribuindo para o conhecimento médico atual e para a indústria farmacêutica, as quais são utilizadas para síntese de vários medicamentos ${ }^{1}$.

A Organização Mundial da Saúde (OMS), desde 1978, estimula seus países membros a reconhecerem o potencial da medicina tradicional e o uso das plantas medicinais e da fitoterapia, como uma prática de autocuidado ${ }^{2}$. Estima-se que $80 \%$ da população mundial utilizam as plantas medicinais. Em 2002 a OMS criou a estratégia sobre medicina tradicional e complementar de forma a auxiliar e apoiar seus membros e autoridades sanitárias a implantar e incentivar políticas e programas que visem aproveitar essa prática de forma segura e efetiva visando ampliar ${ }^{3}$.

- No Brasil, a Política Nacional de Práticas Integrativas e Complementares (PNPIC) configurou um marco para ampliação, reconhecimento e valorização do uso das plantas medicinais no Sistema Único de Saúde (SUS) ${ }^{4}$. O Ministério da Saúde informa que atualmente 2.160 Unidades Básicas de Saúde (UBS) disponibilizam fitoterápicos ou plantas medicinais. Ademais existe o programa Farmácia Viva, que é um programa que compreende todas as etapas, desde o cultivo à distribuição de fitoterápicos no SUS, e se encontra instalado em oitenta municípios da federação ${ }^{6}$.

A utilização de fitoterápicos e plantas medicinais valoriza a cultura e o conhecimento tradicional e o popular; fortalece o desenvolvimento da cadeia produtiva e é uma opção terapêutica aos usuários do SUS ${ }^{4}$.

O presente estudo visa relatar a implantação do canteiro didático de plantas medicinais tem como objetivo recuperar saberes populares tradicionais e discuti-los para 
contribuir com a construção do conhecimento, divulgação de informações e formação ${ }^{5}$ em unidade básica de saúde do Distrito Federal.

\section{MÉTODO}

Relato de experiência sobre a implantação do canteiro medicinal em uma Unidade Básica de Saúde 10 (UBS) do Distrito Federal, realizado por uma residente do Programa de Residência Médica de Medicina de Família e Comunidade da Universidade de Brasília.

A Unidade Básica de Saúde 10 em Ceilândia tem como área de abrangência a população que mora na Ceilândia Norte, realizando atendimentos eletivos programados e atendendo à demanda espontânea dessa comunidade6.

O processo de implantação do canteiro medicinal contou com um grupo de trabalho composto pela residente em Medicina de Família e Comunidade, uma terapeuta ocupacional e uma assistente social, essas pertencentes ao NASF local. A implantação foi realizada no período de agosto a dezembro de 2019.

O estudo foi desenvolvido em duas etapas. A primeira contou com a formação de um grupo de trabalho, sendo realizadas seis reuniões presenciais, sala de reuniões da UBS, tendo em média duração de uma hora. Os objetivos foram: organização da implementação do projeto e confecção de materiais didáticos (formulário de interesse, projeto de implantação e roteiro estruturado), que se encontram em anexo. A segunda etapa refere-se à execução da implantação do projeto.

O plano estratégico para organização e implantação do canteiro medicinal encontra-se apresentado na Tabela 1.

Tabela 1. Plano estratégico para implantação do canteiro medicinal 


\begin{tabular}{|c|c|}
\hline Reuniões & Atividades \\
\hline $1^{\mathrm{a}}$ & $\begin{array}{l}\text { Formação do grupo de trabalho; } \\
\text { Planejamento de trabalho; } \\
\text { Definição do número de integrantes responsáveis pelo projeto; } \\
\text { Definição das funções e responsabilidades de cada integrante; } \\
\text { Criação do formulário de interesse de participação. }\end{array}$ \\
\hline $2^{a}$ & $\begin{array}{l}\text { Buscar parcerias com instituições público e/ou privadas para } \\
\text { implantação do projeto: }\end{array}$ \\
\hline $3^{a}$ & $\begin{array}{l}\text { Buscar parcerias com instituições público e/ou privadas para } \\
\text { implantação do projeto: }\end{array}$ \\
\hline $4^{a}$ & Definição do nome do projeto; \\
\hline $5^{\mathrm{a}}$ & $\begin{array}{l}\text { Planejamento de funcionamento do grupo participante do } \\
\text { projeto; }\end{array}$ \\
\hline $6^{\mathrm{a}}$ & $\begin{array}{l}\text { Avaliação do número de interessados em participar do grupo do } \\
\text { projeto através dos formulários de interesse; }\end{array}$ \\
\hline
\end{tabular}

\section{RESULTADOS}

A implantação do canteiro medicinal contou com a realização de quatro encontros, realizados uma vez por semana, entre novembro e dezembro/2019. Os participantes iniciais do grupo eram servidores e pacientes que preencheram formulário de interesse (em anexo) distribuído na UBS.

O primeiro encontro ocorreu no dia 29/11/19 na UBS10 em Ceilândia quando foi realizada uma dinâmica em grupo, iniciada com a apresentação dos participantes seguida de uma roda de conversa. Foram trazidas questões sobre o interesse dos participantes em conhecer mais sobre plantas medicinais, sobre a PNPMF e sobre a diferença entre os termos "planta medicinal”, "droga vegetal”, “chá medicinal” "fitoterápico", "medicamento fitoterápico", "fitoterápico industrializado e fitoterápico manipulado", segundo as definições da Agência Nacional de Vigilância Sanitária 
(ANVISA)11. Na sequência, foi discutido sobre a camomila (Matricaria recutitta), seu uso tradicional, racional e seguro, segundo referências cientificas do Memento Fitoterápico da Farmacopeia Brasileira de 2016. Finalizou-se o encontro com o plantio das mudas de camomila pelos participantes, com orientações de seu manejo e cultivo. Por fim, foi distribuída sementes registradas da espécie e confecção de um vaso autoirrigável para o plantio das mudas e sementes. Foram utilizadas garrafas de plástico reciclada para acondicionamento das sementes. Foi ressaltado o potencial da reciclagem e reutilização de matérias não degradáveis como instrumentos para cultivo das plantas. Os demais encontros seguiram ocorrendo semanalmente, conforme modelo do primeiro, e estão resumidos na dinâmica de encontros do projeto de implantação que se encontra no apêndice II deste trabalho.

Foi estabelecida uma importante parceria com professores farmacêuticos da Universidade de Brasília- UnB Campus Ceilândia que resultou em parte do quarto encontro do grupo.

Desde a formação do primeiro grupo foi constatado o grande interesse por parte dos profissionais das equipes de saúde local em participarem do projeto e conhecer mais profundamente sobre o uso das plantas medicinais como uma alternativa às técnicas tradicionais da medicina, com o intuito principal de disseminar o conhecimento adquirido e melhor orientar a comunidade. Isso ficou bem evidente no número de registros no formulário de interesse e do perfil dos participantes do grupo que contou com a presença das seguintes categorias profissionais: técnico em saúde bucal, dentista, agente comunitário de saúde, enfermeiros, médicos, vigilantes, técnico em enfermagem, terapeuta ocupacional, nutricionista e assistente social.

\section{DISCUSSÃO}

A apresentação in vivo e in situ das espécies medicinais, o manejo do canteiro e das plantas, as rodas de conversa e os workshops oferecidos durante a execução do projeto 
proporcionaram uma experiência terapêutica aos profissionais envolvidos conforme relato verbal dos participantes.

Um empecilho apresentado foi o de não dispor de mudas registradas oficialmente para distribuição para os participantes dos grupos, uma vez que estas deveriam ter uma avaliação etnobotânica para garantir a espécie a ser utilizada. A solução encontrada para esse problema foi utilizar sementes registradas botanicamente, de forma a garantir a segurança da identificação e uso da planta medicinal.

Lopes et $\mathrm{al}^{7}$, relata em sua experiência de criação do canteiro medicinal questões semelhantes a esse estudo. Foram observadas dúvidas dos profissionais de saúde quanto a credibilidade de princípios ativos e sobre o conhecimento popular e científico a respeito das plantas medicinais. Nesse trabalho foi visto a necessidade de desmitificar as dúvidas sobre o tema, comuns também entre os profissionais de saúde.

Um relato de experiência de um curso de capacitação sobre plantas medicinais para profissionais de nível superior da atenção primária à saúde concluiu que, dos $88 \%$ dos profissionais que trabalham na estratégia da saúde da família, apenas 31\% relataram que orientam a comunidade sobre o uso de plantas medicinais com frequência e $75 \%$ nunca realizaram cursos e/ou capacitação sobre terapias complementares ${ }^{8}$. Nesse sentido é muito importante que se tenha profissionais capacitados a orientar o uso seguro das plantas medicinais, pois além de reconhecer equívocos e reparar erros do uso indevido pela população, estes saberiam orientar quanto as interações medicamentosas e toxicidade nas populações envolvidas ${ }^{9}$

Em outro relato de experiência das plantas medicinais e fitoterapia na atenção primária à saúde, os enfermeiros pesquisados informaram que a união do conhecimento popular sobre plantas medicinais associado à prática clínica do profissional de saúde, pode favorecer a adesão da população ao tratamento devido a aproximação de culturas e valorização do saber popular ${ }^{10}$. A integração a assistência em saúde às práticas de sabedoria popular já reconhecidas no meio científico fazem parte da atribuição da atenção 
primária, pois implicam na aceitação do saber do outro, na valorização da prática de autocuidado pelo usuário e proporciona uma maior aproximação médico-paciente ${ }^{11}$.

Uma pesquisa realizada em Petrolina-PR, sobre utilização popular das plantas medicinais nos clubes de mães, evidenciou em seus resultados que a comunidade, apesar de possuir conhecimentos acerca da utilização as plantas, ainda carece de informações que a orientem sobre o uso seguro e propriedades medicinais das plantas ${ }^{12}$.

Outro potencial dessa prática, é a aproximação do profissional de saúde com a pessoa em cuidado, durante a prática do plantio, compartilhando das potencialidades e dificuldades do processo e estimulando a valorização do meio ambiente. Em algumas outras experiências, isso também foi visto como no Jardim Botânico de Diadema-SP, onde a implantação de canteiros com as espécies vegetais e sua visitação foram uma forma de aproximar os diversos atores sociais ao meio ambiente ${ }^{13}$.

\section{CONCLUSÕES}

É possível considerar que a criação do canteiro medicinal didático proporcionou a criação de um espaço onde possam ser discutidos vários temas de relevância para saúde do indivíduo e de estímulo ao autocuidado. Os programas ou modelos mais estruturados são escassos para implantação e incentivo do uso de plantas medicinais no SUS.

Este relato de experiência evidenciou que os profissionais de saúde desejam e carecem de incentivo para se capacitarem no conhecimento das plantas medicinais e estes são potenciais multiplicadores desse conhecimento.

Este trabalho limitou-se a um trabalho experimental com um prazo de conclusão previamente estabelecido e que por este motivo não alcançou todas as potencialidades que pode vir a apresentar se aperfeiçoado na particularidade de cada estabelecimento de saúde. Sugere-se criar um instrumento de avaliação da atividade para melhor averiguar as lacunas existentes e potencialidades do mesmo. 


\section{Declaração de interesses:}

Os autores declaram não ter conflito de interesse.

\section{Contribuições de cada autor:}

YPCG, FHA, RRDR participaram igualmente do desenho de estudo, da redação do texto, da coleta, da análise e da interpretação dos dados. LVR, IK, JSL participaram da redação do texto e da interpretação dos dados.

\section{Referências}

1. CECHINEL-FILHO, V.; YUNES, R.A. Plantas medicinais sob a ótica da química medicinal moderna. In: YUNES, R.A.; CALIXTO, J.B. (ed.). Chapecó: Argos Ed., Universitária, 2001. 524 p.

2. Organização Mundial da Saúde - OMS. Cuidados primários da saúde. Relatório da Conferência Internacional sobre Cuidados Primários de Saúde Alma-Ata, URSS, 6-1 2 de setembro de 1978. UNICEF. Brasil, 1979.

3. Organização Mundial da Saúde - OMS. Estrategia de la OMS sobre medicina tradicional $2014 \quad$ - 2023.20 Disponível <http://www.who.int/about/licensing/copyright_form/en/index.html>. Acesso: 28 de nov. de 2019.

4. Brasil. Ministério da Saúde. Secretaria de Atenção à Saúde. Departamento de Atenção Básica. Política Nacional de Práticas Integrativas e Complementares no SUS - PNPICSUS- Brasília: Ministério da Saúde, 2006.

5. MARTINS, R.D; PINTO, R. H; SENNA, S. M; LIMA, A. W; MOTA, C. R. DE F DE C; FONTES, D. A. F; BARROS, F. A; XIMENES, R. M. Estruturação do Espaço 
Farmácia Viva na Universidade Federal de Pernambuco como Estratégia para Formação em Fitoterapia. Vittalle - Revista de Ciências da Saúde v. 30, n. 1, p. 182-191, 2018.

6. Secretaria de Saúde do Distrito Federal. Unidades Básicas de Saúde - Ceilândia. Disponível em: http://www.saude.df.gov.br/ubs-ceilandia/. Acessado em 28 de nov. de 2019.

7. LOPES, Julieta Maria Del; LINK, Dionisio. Implantação de um horto didático de plantas bioativas no município de tupanciretã. Revista Eletrônica em Gestão, Educação e Tecnologia Ambiental, [S.1.], p. 225-250, mar. 2011.

8. CEOlin, T; CEOlin, S; HECKA, R. M; NOGUEZA, P. T; SOUZA, A. D. Z de. Relato de experiência do curso de plantas medicinais para profissionais de saúde. Revista Baiana de Saúde Pública, v.37, n.2, p.501-511 abr./jun. 2013.

9. MADEIRO, Amanda Arruda Santos; Lima, Cristiano Ribeiro. Estudos etnofarmacológicos de plantas medicinais utilizadas no Brasil: Revisão da literatura. Cadernos de graduação- ciências biológicas e da saúde, Maceió, v.3, n.1, p. 69-76, nov. 2015.

10. VARELA, D. S. S; AZEVEDO, D. M. Opinião de médicos e enfermeiros sobre o uso da fitoterapia e plantas medicinais na atenção básica. Rev. APS, v.17, n.2, p.204-213, abr/jun. 2014.

11. ROSA, Caroline da; CAMARA, Sheila Gonçalves; BERIA, Jorge Umberto. Representações e intenção de uso da fitoterapia na atenção básica à saúde. Ciência \& saúde coletiva, Rio de Janeiro, vol.16, n.1, p. 311-318, jan. 2011.

12. PAUlERT, R; PUJARRA, S; OLIVEIRA, F. G. DE; ZONETTI, P. DA C; RUPPELT, B. M. Utilização popular de plantas medicinais nos clubes de mães de Palotina-PR. Revista Ciência em Extensão. v.10, n.2, p.55-64, 2014. 
13. CASSAS, F; SILVA, D. S. DA; CONSUElO BARROS, C; REIS, N. F. DE C; RODRIGUES, E. Canteiros de plantas medicinais, condimentares e tóxicas como ferramenta de promoção à saúde no jardim botânico de Diadema, SP, Brasil. Revista Ciência em extensão. v.12, n.2, p.37-46, 2016.

\section{APÊNDICE I: RESUMO DO FORMULÁRIO DE INTERESSE EM PARTICIPAÇÃO DO GRUPO O CANTEIRO MEDICINAL}

O que será este grupo?

Quem poderá participar?

Quando ocorrerá?

O que será desenvolvido no grupo?

Se você tem interesse na participação deste grupo, deixe seu nome

\section{APÊNDICE II: DINÂMICO DE ENCONTROS DO PROJETO DE IMPLANTACAO DO CANTEIRO MEDICINAL}

\begin{tabular}{|c|l|}
\hline 1 E Encontro & $\begin{array}{l}\text { Apresentação do grupo: } \\
\text { Dinâmica: De onde vem o interesse dos participantes pelo tema? } \\
\text { Roda de Conversa: } \\
\text { Tema1: Apresentação do projeto e cronograma das atividades } \\
\text { Planta em foco: } \\
\text { Matricaria Recutitta (Camomila): princípios ativos, formas de uso, uso seguro } \\
\text { Prática: } \\
\text { Características e noções básicas do cultivo desta espécie; } \\
\text { Limpeza e adubação dos espaços, plantio das mudas pelos participantes do do uso etc. }\end{array}$ \\
\end{tabular}




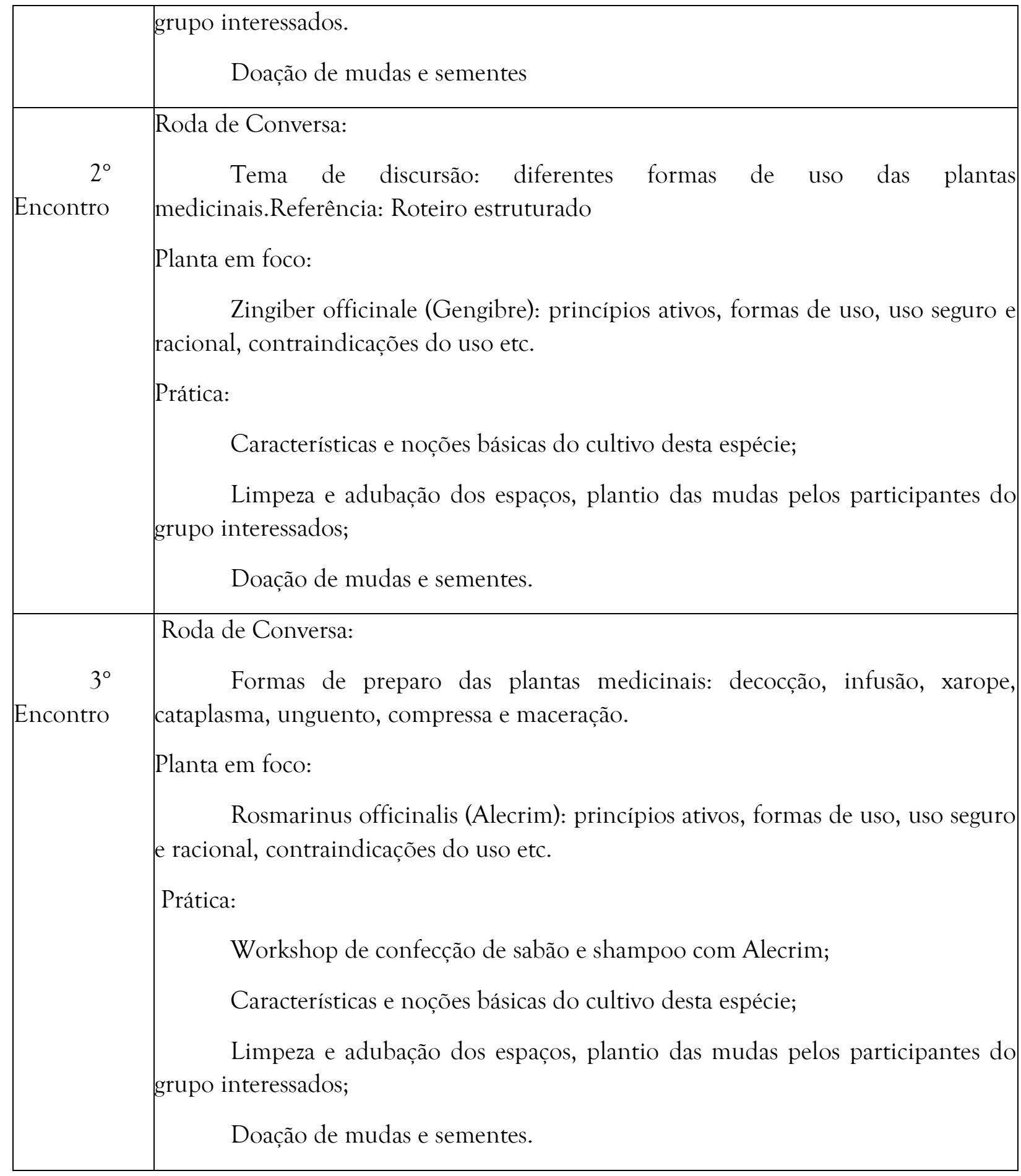




\begin{tabular}{|c|l|}
\hline $4^{\circ}$ & $\begin{array}{l}\text { Visita ao horto didático da UNB campus Ceilândia } \\
\text { Encontro }\end{array}$ \\
$\begin{array}{l}\text { Dinâmica organizada pelos professores e alunos do campus. } \\
\text { Planta em foco: } \\
\text { Calendula oficinales (Calêndula):princípios ativos, formas de uso, uso seguro } \\
\text { Prática: } \\
\text { Limpeza e adubação dos espaços, plantio das sementes colhidas no horto } \\
\text { didático da UNB campus Ceilândia; } \\
\text { Doação de sementes. }\end{array}$ \\
\hline
\end{tabular}

\section{APÊNCIDE III: RESUMO DO ROTEIRO ESTRUTURADO}

Por que falar de plantas medicinais?

O que o Ministério da Saúde diz a respeito do tema?

O que é: Planta medicinal; Droga Vegetal, Chá Medicinal, Fitoterápico, Medicamento fitoterápico; fitoterápico manipulado; fitoterápico industrializado?

Quais as formas de usar as plantas medicinais?

Quais as maneiras de preparar a planta medicinal?

Cuidados na utilização das plantas medicinais;

Noções básicas de cultivo e propagação das plantas medicinais;

Colheita, secagem e armazenamento das plantas medicinais. 